\title{
La question des langues chez Élie Wiesel
}

\section{Michaël de Saint-Cheron}

\section{(2) OpenEdition}

Journals

Édition électronique

URL : https://journals.openedition.org/tsafon/558

DOI : $10.4000 /$ tsafon.558

ISSN : 2609-6420

\section{Éditeur}

Association Jean-Marie Delmaire

\section{Édition imprimée}

Date de publication : 1 juin 2018

Pagination : 35-42

ISSN : 1149-6630

\section{Référence électronique}

Michaël de Saint-Cheron, « La question des langues chez Élie Wiesel », Tsafon [En ligne], 75 | 2018, mis en ligne le 06 mai 2019, consulté le 30 juin 2021. URL : http://journals.openedition.org/tsafon/558 ; DOI : https://doi.org/10.4000/tsafon.558 


\title{
La question des langues chez Élie Wiesel
}

\author{
Michaël de Saint-Cheron*
}

À la mémoire de Claude Hampel '"ז

Il y a une question des langues chez Élie Wiesel comme chez plusieurs écrivains et poètes de l'exil en général et en particulier pour les survivants des grandes tragédies de l'histoire comme Aharon Appelfeld, Piotr Rawicz parmi beaucoup d'autres juifs ou non-juifs, au siècle dernier en particulier.

La langue courante au shtetl était le yiddish, la langue religieuse était l'hébreu, et les enfants entendaient réciter les prières avant leur venue au monde, mais à Sighet, la langue officielle fut tour à tour le hongrois et le roumain, et certains paysans parlaient même le ruthène et le russe. Élie Wiesel écrira longtemps plus tard un véritable hymne à la langue de son enfance :

Il est des chants que je ne peux chanter qu'en yiddish. Il est des prières que seules les grand-mères juives avaient coutume de chuchoter dans la pénombre du crépuscule. [...] Il est des contes dont seule la langue yiddish, inondée de tristesse et de nostalgie, peut rendre la magie et le mystère. ${ }^{1}$

Les psychanalystes parlent de transfert. Y a-t-il donc eu transfert d'une langue à l'autre chez notre écrivain-témoin ? Remarquons toutefois que les écrivains changeant de langue ont dans la majorité des cas adopté celle de leur pays d'accueil devenue leur langue d'élection. Dans le cas

\footnotetext{
* École Pratique des Hautes Études, Paris.

${ }^{1}$ «Rand marshoves vegn yiddish», Di goldene keyt $\mathrm{n}^{\circ} 123,1987$, Tel-Aviv, trad. du yiddish par Rachel Ertel, cité dans Autour d'Élie Wiesel. Une parole pour l'avenir, colloque de Cerisy (sous la dir. de Michaël de Saint-Cheron), Paris, Odile Jacob, 1996, p. 21-40.
} 
de Wiesel, les choses sont moins simples car si le français est bien la langue du pays qui l'accueillit à son retour des camps, elle n'est pas celle du pays où il s'installa pour vivre. S'il opta pour le français, c'est d'abord pour Mauriac. Et puis sa connaissance de l'anglais, qui était parfaite à l'oral, ne lui permettait pas, selon ses dires, d'écrire directement dans la langue. Pourtant, Wiesel aurait fort bien pu conserver le yiddish pour écrire, ses combats, sa notoriété ne l'eurent sans doute pas moins distingué en 1986 pour le Nobel de la paix, puisque Isaac Bashevis Singer ne s'en départit jamais, ce qui ne l'empêcha pas de recevoir le Nobel de littérature en 1978 et, dans son discours de réception du prix, il déclara :

Les fantômes aiment le yiddish et, pour autant que je sache, ils le parlent tous. Je ne crois pas seulement aux démons et aux autres esprits, mais aussi à la résurrection. Je suis sûr qu'un jour, des millions de cadavres parlant yiddish se lèveront de leurs tombes, et la première question qu'ils poseront sera : quel est le dernier livre paru en yiddish ? ${ }^{2}$

Pourquoi alors avoir choisi cette langue, le français, si éloignée du yiddish comme de l'hébreu ? Je ne reviendrai pas sur François Mauriac. Mais Wiesel aurait pu continuer à écrire en yiddish le reste de ses livres, or il n'écrivit en yiddish que des articles et quelques textes plus essentiels pour la presse de la Yiddishkeit américaine ou israélienne comme $D i$ goldene keyt, publiée à Tel-Aviv. La deuxième réponse est sans doute de même nature que celle que l'on pourrait donner à la question de savoir pourquoi il choisit finalement de s'installer aux États-Unis, à New York, plutôt qu'en France ou qu'en Israël. Le français est d'abord une langue plus lue, donc plus internationale que le yiddish. La troisième réponse, celle qu'il apporte lui-même, est beaucoup plus métaphysique, en ce qu'elle intègre l'être et l'au-delà de l'être, la mémoire et l'au-delà de la mémoire, c'est-à-dire une mémoire transfigurée :

Après la guerre, j'avais adopté cette langue avec un réel sentiment d'amour. Mais cette langue, en principe, ne devrait pas être la mienne car elle ne convient pas toujours aux thèmes que je traite dans mes livres. [...] Choisir le français relevait du défi, je crois. Je cherchais également une langue différente : l'hébreu, le yiddish ou le hongrois symbolisaient toute mon enfance. [...] Et je voulais montrer que j'étais entré dans une ère nouvelle, pour me prouver à moi-même

\footnotetext{
${ }^{2}$ Cf. www.huffingtonpost.fr/edith-ochs/langue-yiddish-litterature_b_2717800.html
} 
que j'étais vivant, que j'avais survécu. Je tenais à rester le même, mais dans un autre paysage. Et la langue française constituait ce nouveau paysage. ${ }^{3}$

De toutes les langues parlées d'Élie Wiesel, on peut dire que deux langues au sens fort furent pour lui langue d'élection: le français et le yiddish, sa langue maternelle. L'hébreu fut celle que les Wiesel parlaient lors du shabbat à la maison à Sighet et lui fut une chance dès 1949, quand il toucha la terre d'Israël et en revint correspondant du Yedioth aharonot. L'anglais est devenu une langue de travail puis celle de sa vie aux États-Unis et de son enseignement. Alors que le français fut non seulement sa langue littéraire mais aussi celle de son foyer avec Marion, sans parler de ses amitiés françaises. Mais reprenons le texte capital traduit par Rachel Ertel pour le colloque que j'avais dirigé à Cerisy-laSalle, en juillet 1995, qui reste une fierté car il fut l'unique colloque sur l'œuvre d'Élie tenu en France en sa présence :

Le yiddish, ce sont mes années d'enfance. Le yiddish est un royaume en soi... Les mots sont comme les hommes : il en est de riches et de pauvres, de petits et de grands, de majestueux et de boursouflés, il en est de vieux et voûtés, comme il en est de jeunes et altiers. Essayez donc, prenez une phrase, analysez-la, approfondissez-la, et vous révélerez des générations de Juifs, venus de toutes les contrées de l'exil. Vrai, on peut en dire autant d'autres langues. Mais montrezmoi un peuple qui ait porté avec lui le joug de tant de saintetés, de lieu en lieu, de pays en pays...

Le yiddish est bon pour le rire et les larmes. Pour des situations neutres, il faut chercher d'autres langues. Se pose alors une question ardue: existe-t-il des situations neutres pour les Juifs? [...] Depuis la chute du second Temple, les Juifs vivent toujours dans des situations extrêmes. Personne ne porte un tel fardeau de tristesse, personne ne combat la tristesse avec autant de persévérance, avec autant de joie...

Parfois je n'arrive pas à concevoir comment des Juifs, dans l'épreuve ultime [...] parvenaient, au plus profond de l'abîme, dans la nuit même d'Auschwitz, à entonner les mélodies du Shabbat, quand la mort se tapissait dans chaque trou et les guettait, quand les flammes serpentaient vers le Temple céleste...

Et puisque nous parlons de ces temps de ténèbres et de nuit, il faut peut-être souligner qu'il n'est pas dans le monde de langue pour les évoquer pareille au yiddish. La littérature de l'Anéantissement serait sans le yiddish comme sans âme. Je sais, on écrit aussi en d'autres langues. Mais on ne peut le comparer. Les œuvres les plus authentiques sur l'Anéantissement, en prose comme en poésie, sont en yiddish. Est-ce parce que la plupart des victimes sont issues de cette langue et y ont vécu ? Que les professionnels répondent à cette question.

\footnotetext{
${ }^{3}$ Brigitte-Fanny Cohen, Élie Wiesel, qui êtes-vous?, Lyon, La Manufacture, 1987, p. $72-73$.
} 
Quant à moi, je ne sais qu'une chose : s'il n'y avait eu mon premier livre en yiddish, s'il n'y avait eu mes mémoires en yiddish, mes autres livres auraient sombré dans la mutité. ${ }^{4}$

Alors oui, le yiddish restait pour lui « La » langue par excellence où l'on pouvait parler de l'Extermination. D'où la question que nous reposons : pourquoi a-t-il finalement opté malgré tout pour le français alors qu'il écrivait le yiddish comme sa langue maternelle ? Il y a eu $L a$ Nuit, mais est-ce que ce livre fut celui qui lui a fait choisir finalement, qui l'a fait opter pour une langue universelle, laissant le yiddish pour des textes à la marge de son œuvre, sans doute les plus intimes de ceux qu'il ait écrits?

Pour lui, les voix capitales qui ont parlé de la Shoah, qui se dit Hurban en yiddish, du mot hébreu qui désigne la destruction du Temple, sont celles des poètes et écrivains yiddish, dont beaucoup nous sont parvenus comme œuvres ou témoignages posthumes. Le Chant du peuple juif assassiné d'Ytzhok Katzenelson, mort à Birkenau avec son fils le $1^{\text {er }}$ mai 1944 après avoir réussi à échapper à l'extermination du ghetto de Varsovie, est parmi les chefs-d'œuvre poétique sur la Catastrophe. Mais comment lire l'affirmation de Wiesel face aux poèmes tout simplement hantés de Paul Celan écrits en allemand, mais dans une langue nouvelle ? Qui d'autre que Celan parla non pas de l'absence de Dieu ou de son silence mais du dieu absent de la Shoah comme du Niemand [Personne] dans son terrible poème Psalm (Psaume) : "Gelobt seist du, Niemand (Loué sois-tu, Personne) ». ${ }^{5}$

À mon sens, une autre langue, voire deux autres langues, sont sousjacentes à toute l'œuvre de Wiesel : celle du silence, celle du chant, qui sont coextensives à sa parole. J'ai même envie d'aller loin dans les paradoxes : la langue de « Sa » prière est aussi une langue à part, à nulle autre pareille.

Marquant la place du silence, du silence de la langue, des langues, à dire jusqu'au bout l'indicible - même si pour Jorge Semprún, il n'y a pas d'indicible même sur les camps - Wiesel soulignait la part d'irréductible, d'inexprimable, à la façon dont Schönberg fait dire à Moïse à la toute fin de son ultime opéra Moses und Aron : «O Wort, du Wort, das mir fehlt ! [Ô Verbe, toi qui me manque !] ».

\footnotetext{
${ }^{4}$ Autour d'Élie Wiesel. Une parole pour l'avenir, op. cit., p. 22-23.

${ }^{5}$ Paul Celan, «Psalm», Die Niemandsrose. Sprachgitter, Frankfurt a. M., Fischer Taschenbuch Verlag, p. 26.
} 
Si le français est certes plus rationnel que le yiddish et sans doute moins métaphysique que l'allemand - cela est une grave question quand on voit les tenants de la métaphysique allemande depuis des siècles ! - il peut aussi s'amuïr, s'affoler, se faire mystique, sortir de ses gonds, mais le yiddish, j'y reviendrai, a une supériorité à nulle autre seconde, il est la langue des morts, la langue d'un monde disparu, la langue que les tueurs ont assassinée - et ces faits lui donnent une puissance d'effroi, une force térébrante, à travers aussi la dérision, qui ne se compare à rien. " La parole ne doit être ni emprisonnée ni jugulée, pas même par le silence de la page ; il faut la libérer pour la capter », écrit-il dans Tous les fleuves vont à la mer ${ }^{6}$. Pourtant, une page plus haut, il interrogeait encore et toujours :

Peut-on parler du silence de Dieu autrement que par le silence humain ? [...] Derrière chaque mot, il y a d'autres mots. Et derrière eux, d'autres mots encore, et d'autres, tous intangibles, invisibles, mais chargés d'attente et d'anticipation. ${ }^{7}$

Qui se souvient dans La Nuit des deux prières terribles qu'il prononce - au moins dans son souvenir - le jour de Rosh Hashana 1944 à Buna?

Qu'es-Tu, mon Dieu, pensais-je avec colère, comparé à cette masse endolorie qui vient Te crier sa foi, sa colère, sa révolte ? Que signifie Ta grandeur, maître de l'Univers, en face de toute cette faiblesse, en face de cette décomposition et de cette pourriture? Pourquoi encore troubler leurs esprits malades, leurs corps infirmes?

Dix mille hommes étaient venus assister à l'office solennel, chefs de block, kapos, fonctionnaires de la mort. [...]

Béni soit le nom de l'Éternel!

Des milliers de bouches répétaient la bénédiction, se prosternaient comme des arbres dans la tempête.

Béni soit le nom de l'Éternel!

Pourquoi, mais pourquoi le bénirais-je? Toutes mes fibres se révoltaient. Parce qu'Il avait fait brûler des milliers d'enfants dans ses fosses? Parce qu'Il faisait fonctionner six crématoires jour et nuit les jours de Shabbat et les jours de fête? Parce que dans Sa grande puissance Il avait créé Auschwitz, Birkenau, Buna et tant d'usines de la mort? Comment Lui dirais-je : «Béni sois-Tu, l'Éternel, Maître de l'Univers, qui nous as élus parmi les peuples pour être torturés jour et nuit, pour voir nos pères, nos mères, nos frères finir au crématoire ? Loué soit Ton Saint Nom, Toi qui nous as choisis pour être égorgés sur Ton autel ?» [... ] Et moi, le mystique de jadis, je pensais :

\footnotetext{
${ }^{6}$ Élie Wiesel, Tous les fleuves vont à la mer, Paris, Seuil, 1996 (Points), p. 457.

${ }^{7}$ Ibid, p. 457-458.
} 
«Oui, l'homme est plus fort, plus grand que Dieu. Lorsque Tu fus déçu par Adam et Ève, Tu les chassas du paradis. Lorsque la génération de Noé Te déplut, Tu fis venir le Déluge. Lorsque Sodome ne trouva plus grâce à Tes yeux, Tu fis pleuvoir du ciel le feu et le souffre. Mais ces hommes-ci que Tu as trompés, que $\mathrm{Tu}$ as laissés torturer, égorger, gazer, calciner, que font-ils ? Ils prient devant Toi ! Ils louent Ton nom ! ${ }^{8}$

Pour moi ce texte et ceux qui sont de la même famille, nés de la protestation infinie, du refus infini de croire en un Dieu qui laisse faire cela, sont à la fois transposables en hébreu, yiddish, français. Ils viennent du yiddish, Wiesel les a traduits en français puis les a réécrits en hébreu pour la troisième version d'Un di velt hot geshwign... [Et le monde se taisait] ${ }^{9}$.

Alors pourquoi, oui pourquoi n'a-t-il pas rompu avec toute forme de foi, lui dont la foi agonique, totalement tragique et totalement sans réponse, avait atteint un paroxysme de douleur et d'incompréhension ? Sans doute parce que le refus définitif de toute pratique religieuse eût été à ses yeux, plus tragique encore que son combat intérieur entre un Dieu mort ou muet à jamais et un non-Dieu ? Son attachement à ce Dieu muet a quelque chose de bouleversant, ce Dieu muet dont parle déjà le Talmud par la bouche de Rabbi Ismaël, qui s'écria: "Mi kamokha baélim adoshem, al tikra élim ki im ilémim ki roé beelbon banav veshotek [Qui est comme toi parmi les dieux ? Qui donc est aussi muet que toi puisque tu vois l'humiliation de tes enfants et tu te tais ?] » ${ }^{10}$. Car s'il y a non-Dieu, alors pourquoi avoir été juif? Alors pourquoi être resté Juif? Alors pourquoi avoir perduré comme peuple juif dans la longue histoire tragique et incomparable qui fut celle de nos ancêtres? Ici, George Steiner apporte une réponse que je ne peux taire tant je la fais mienne avec beaucoup - avec tant d'autres :

Si, dans la Passion du Christ, un être divin, un Fils de Dieu et de l'Homme, est censé être mort pour l'homme, ainsi, dans la Shoah, le peuple juif (« Radix, Matrix ») [...] peut être vu, compris, comme étant mort pour Dieu, comme ayant pris sur lui l'inconcevable culpabilité de l'indifférence, ou de l'absence, ou de l'impuissance de Dieu. ${ }^{11}$

\footnotetext{
${ }^{8}$ Élie Wiesel, La Nuit, Paris, Éditions de Minuit, 1958/2007, p. 126-127.

${ }^{9}$ Titre de la première parution, en yiddish, de La Nuit.

${ }^{10}$ Élie Wiesel, Paroles d'étranger, Paris, Seuil, 1982, p. 177 (Traité Guittin 56 b).

${ }^{11}$ George Steiner, «La longue vie de la métaphore. Une approche de la Shoah », trad. de l'anglais par Marie Moscivici, L'Écrit du temps, $\mathrm{n}^{\circ}$ 14/15, Paris, Éditions de Minuit, 1987, p. 15-33.
} 
Qui a dit quelque chose de plus effrayant mais de plus juste aussi ?

La réponse de Wiesel est dans le chant. C'est pourquoi, selon une formulation talmudique connue, je dirais : quand toutes les portes de la raison et de la foi et du cœur sont fermées, celle du chant restait opiniâtrement ouverte pour l'écrivain hassid écartelé qu'il fut par le vide de Dieu. C'est pourquoi, l'œuvre quasi théologique de Wiesel me semble toujours l'une des plus capitales du siècle dernier et de notre début de $\mathrm{XXI}^{\mathrm{e}}$ siècle, né dans la terreur et l'horreur non plus des exterminations de masse mais dans celles du terrorisme, du fanatisme au nom d'Allah! Peut-être aussi au nom d'autres dieux, comme le dieu Capital, comme le dieu Puissance, comme le dieu Pétrole mais aussi comme tous les petits dieux qui poussent les hommes et les femmes du monde entier à tuer, à combattre au nom de leur croyance.

La langue d'Élie Wiesel est le doute infini et le doute a besoin des langues saintes comme l'hébreu, comme il a besoin de se dire dans la langue du peuple assassiné, du peuple anéanti par les nazis, par l'Allemagne nationale-socialiste et ses sbires, comme il a besoin de trouver assise dans une troisième langue qui n'est plus héritière d'une transcendance, qui n'est plus héritière d'un peuple disparu, mais qui est l'héritière d'une culture deux fois millénaire, d'une littérature bien moins ancienne que la Torah et le Talmud, mais qui a conquis le monde depuis près de dix siècles et plus encore depuis Rabelais, Pascal, Descartes, Montaigne jusqu'à Victor Hugo, jusqu'à Proust, Aragon, Camus, Malraux puis jusqu'à Bergson, Levinas, Derrida et nos contemporains. La langue d'Élie Wiesel est faite de toutes ces langues incorporant le silence, la protestation métaphysique et religieuse sans jamais oublier la part du chant, qui unifie et fait converger tous les contraires. 
Maison natale d'Élie Wiesel

Sighet, 2010

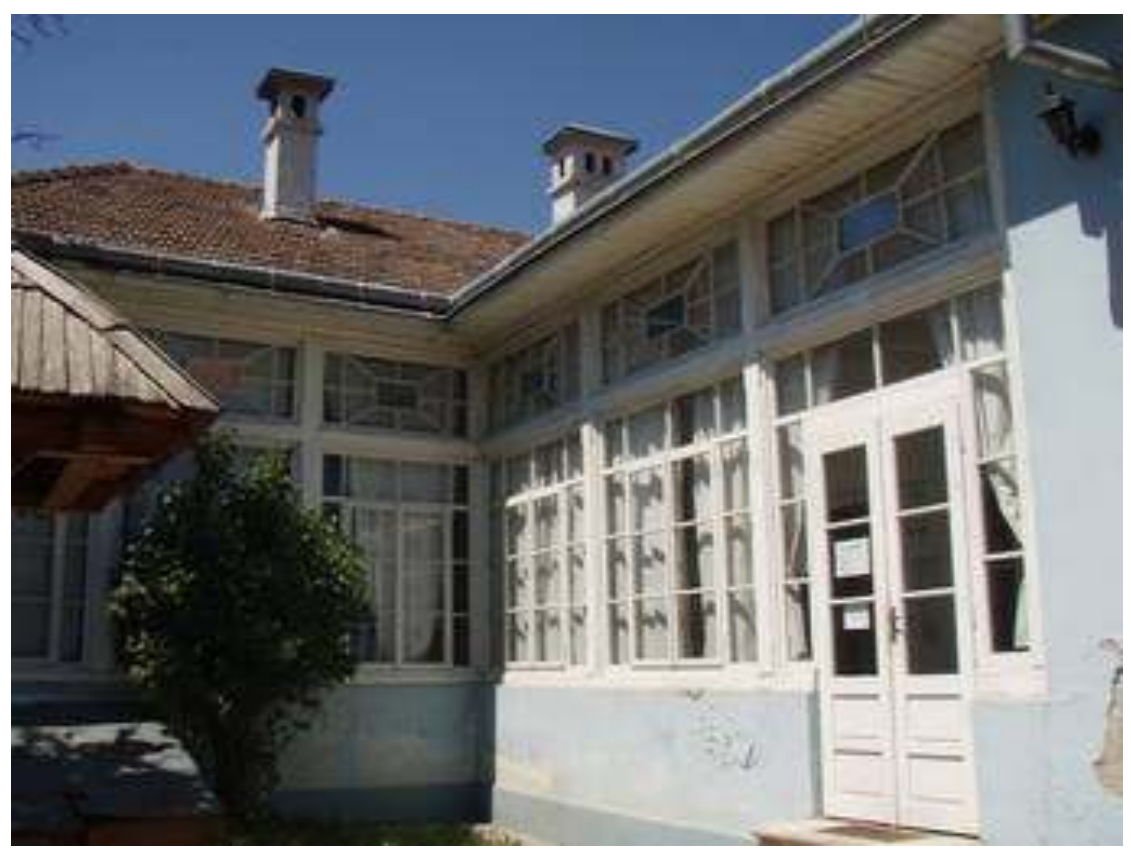

En 2010, la maison n'était pas visitable et le jardin attenant était mal entretenu

Photo : Miriam Panigel

Consulter le site internet : http://miriampanigel.blog.lemonde.fr/

Voir Monique Heddebaut, « Un voyage vers Élie Wiesel, l'oublié », Tsafon $\mathrm{n}^{\circ}$ 64, automne 2012 - hiver 2013, p. 121-125

(Page de la rédaction) 